\title{
High throughput quantitative reverse transcription PCR assays revealing over-expression of cancer testis antigen genes in multiple myeloma stem cell-like side population cells
}

\author{
Jianguo Wen ${ }^{1}$, Hangwen $\mathrm{Li}^{2}$, Wenjing Tao ${ }^{3}$, Barbara Savoldo ${ }^{4}$, Jessica A. Foglesong ${ }^{5}$, \\ Lauren C. King ${ }^{1}$, Youli Zu ${ }^{1}$, and Chung-Che Chang ${ }^{6,7}$ \\ ${ }^{1}$ Department of Pathology and Genomic Medicine, Houston Methodist Hospital, Houston, TX \\ ${ }^{2}$ Department of Urology, Comprehensive Cancer Center, University of Michigan, Ann Arbor, MI \\ ${ }^{3}$ Department of Translational Molecular Pathology, University of Texas MD Anderson Cancer \\ Center, Houston, TX \\ ${ }^{4}$ Department of Pediatrics, Section of Hematology-Oncology, Baylor College of Medicine, \\ Houston, TX \\ ${ }^{5}$ Department of Pediatrics, University of Texas MD Anderson Cancer Center, Houston, TX \\ ${ }^{6}$ Department of Pathology, University of Central Florida, Orlando, FL \\ ${ }^{7}$ Hematology and Molecular Pathology, Department of Pathology, Florida Hospital, Orlando, FL, \\ USA
}

\section{Summary}

\begin{abstract}
Multiple myeloma (MM) stem cells, proposed to be responsible for the tumourigenesis, drug resistance and recurrence of this disease, are enriched in the cancer stem cell-like side population (SP). Cancer testis antigens (CTA) are attractive targets for immunotherapy because they are widely expressed in cancers but only in limited types of normal tissues. We designed a high throughput assay, which allowed simultaneous relative quantifying expression of 90 CTA genes associated with MM. In the three MM cell lines tested, six CTA genes were over-expressed in two
\end{abstract}

\footnotetext{
(C) 2014 John Wiley \& Sons Ltd

Correspondence: Dr Youli Zu, Department of Pathology and Genomic Medicine, Houston Methodist Hospital, Houston, TX 77030, USA. yzu @ houstonmethodist.org and Dr Chung-Che Chang, Department of Pathology, University of Central Florida, and Hematology and Molecular Pathology, Department of Pathology, Florida Hospital, Orlando, FL 32803, USA.

C.Jeff.Chang.MD@Flhosp.org.

Competing interest

The authors declare no competing financial interests.

Author contribution

JW, HL, and WT performed the research. JW, HL, WT, and LK designed the research and the experiments and analysed the data. CC and $\mathrm{YZ}$ supervised the research and provided funding. JF and BS discussed the results. JW wrote the manuscript. All authors read and approved the final manuscript. All authors read and approved the final manuscript.

Supporting Information

Additional Supporting Information may be found in the online version of this article:

Fig S1. Immunofluorescence membrane staining with anti-AURKA antibody in SP and MPC of RPMI8226 cells.

Fig S2. Up-regulated CTA genes in the ALDH high population of MM cell line compared with ALDH low population.

Table SI. MM patient information.

Table SII. Up-regulated CTA genes in SP of MM patient BMs.
} 
and $L U Z P 4$ and $O D F 1$ were universally up-regulated in all three cell lines. Subsequent study of primary bone marrow (BM) from eight MM patients and four healthy donors revealed that 19 CTA genes were up-regulated in SP of MM compared with mature plasma cells. In contrast, only two CTA genes showed a moderate increase in SP cells of healthy BM. Furthermore, knockdown using small interfering RNA (siRNA) revealed that LUZP4 expression is required for colonyforming ability and drug resistance in MM cells. Our findings indicate that multiple CTA have unique expression profiles in MM SP, suggesting that CTA may serve as targets for immunotherapy that it specific for MM stem cells and which may lead to the long-term cure of MM.

\section{Keywords}

multiple myeloma; cancer testis antigen; side population; high throughput; quantitative RT-PCR

Multiple myeloma (MM) is the second most common haematological cancer and represents $10 \%$ of all haematopoietic malignancies in the United States (Jemal et al, 2007). In 2010, the National Cancer Institute reported 20180 new cases of MM and 10650 deaths directly attributed to MM (Cruz et al, 2011). Despite recent major improvements in treatment, MM still remains incurable and long-term survival appears elusive (Chanan-Khan et al, 2010; Borrello, 2012). Current therapy focuses on killing the myeloma cells with cytotoxic agents, targeting myeloma cell-specific pathways, or inhibiting the myeloma-dependent microenvironment. Although these therapies can lead to initial complete clinical response, the majority of patients develop relapsed disease with resistance to these therapies (Munshi et al, 2002; Richardson et al, 2003). This has led to the hypothesis that myeloma stem celllike cells, which have increased resistance to many cytotoxic agents and possess selfrenewal capacity, may be responsible for the relapsed disease (Hajek et al, 2013). Additional methods are required to eliminate the myeloma stem cells for the long-term cure of MM.

Cancer testis antigens (CTA) are a promising class of tumour antigens for T-cell-mediated immunotherapy due to their limited expression in somatic tissue. An earlier study demonstrated that CTA could be specifically recognized in vitro by cytotoxic T-cells (CTLs) in patients with melanoma (van der Bruggen et al, 1991). Since then, more and more CTA genes have been characterized and tested as potential targets for cancer therapy (de Carvalho et al, 2012; Mengus et al, 2013). Furthermore, another recent study showed that CTA genes were highly and frequently expressed in glioma cancer stem cells compared with differentiated cells (Yawata et al, 2010). Encouraged by previous reports, we hypothesized that CTA genes may have a different expression profile in myeloma stem cells compared to mature plasma cells (MPC) and the over-expressed CTA genes may become ideal targets for immunotherapy for the elimination of myeloma stem cells.

In present study, based on gene expression data from MM and normal samples, we designed a high throughput quantitative reverse transcription polymerase chain reaction (qRT-PCR) assay to simultaneously measure the expression of $90 \mathrm{CTA}$ genes associated with $\mathrm{MM}$. We identified several CTA genes whose expressions are increased in the MM SP. Furthermore, we found that one of those up-regulated CTA genes, LUZP4, is required for colony forming 
and drug resistance in MM cells. These findings will lead to the development of new CTLmediated therapies by targeting these CTA genes, for the benefit of MM patients.

\section{Material and methods}

\section{Cell cultures and reagents}

Multiple myeloma cell lines RPMI8226, MM1S and U266 were purchased from the American Type Culture Collection (ATCC; Manassas, VA, USA). Cells were grown in RPMI 1640 medium (Life Technologies, Grand Island, NY, USA) with 10\% fetal bovine serum (FBS; Atlanta Biologicals, Atlanta, GA, USA), $100 \mathrm{u} / \mathrm{ml}$ penicillin and $100 \mu \mathrm{g} / \mathrm{ml}$ streptomycin (Thermo Fisher Scientific, Houston, TX, USA), as previously reported (Wen et $a l, 2011)$.

Bone marrow (BM) aspirates were collected from eight MM patients and four healthy donors under a protocol approved by the Houston Methodist Research Institute and informed consent was obtained, in compliance with the Declaration of Helsinki. Primary cells were purified from freshly isolated BM by Ficoll (MP Biomedicals, Solon, OH, USA) density sedimentation. Cells were cultured in RPMI 1640 medium containing 10\% FBS, 100 $\mathrm{u} / \mathrm{ml}$ penicillin, $100 \mu \mathrm{g} / \mathrm{ml}$ streptomycin and $2 \mathrm{mmol} / \mathrm{l}$-glutamine, and maintained at $37^{\circ} \mathrm{C}$ in $5 \% \mathrm{CO}_{2}$.

All chemicals, unless otherwise stated, were purchased from Sigma-Aldrich Co. (St. Louis, MO, USA).

\section{SP analysis and cell sorting by flow cytometry}

To identify the SP in MM cell lines, cells were seeded at $0.5 \times 10^{6}$ cells $/ \mathrm{ml}$ in a T75 flask for $3 \mathrm{~d}$ (72 h after seeding) in RPMI 1640 medium with 10\% FBS, supplemented with 100 $\mathrm{u} / \mathrm{ml}$ penicillin and $100 \mu \mathrm{g} / \mathrm{ml}$ streptomycin. Cells were harvested and washed in prewarmed RPMI 1640 medium with 2\% FBS and $10 \mathrm{mmol} / \mathrm{l} \mathrm{HEPES} \mathrm{buffer,} \mathrm{and} \mathrm{then}$ resuspended at a concentration $1 \times 10^{6}$ cells $/ \mathrm{ml}$ in RPMI 1640 medium with 2\% FBS and 10 $\mathrm{mmol} / \mathrm{l} \mathrm{HEPES}$. Hoechst 33342 water solution $(1 \mathrm{mg} / \mathrm{ml})$ was then added to make a final concentration of $5 \mu \mathrm{g} / \mathrm{ml}$ followed by incubation in a water bath at $37^{\circ} \mathrm{C}$ for $90 \mathrm{~min}$ with shaking every $15 \mathrm{~min}$. Immediately after incubation, the samples were put on ice to stop dye efflux and washed with ice-cold Hank's balanced salt solution (HBSS) containing 2\% FBS and $10 \mathrm{mmol} / \mathrm{l} \mathrm{HE}-\mathrm{PES}$. Subsequently, Hoechst-labelled cells were stained with CD138-PE monoclonal antibody (BD Biosciences, San Jose, CA, USA) for $15 \mathrm{~min}$ on ice.

To identify the SP in primary samples, cells were harvested and washed in pre-warmed RPMI 1640 medium with 2\% FBS and 10 mmol/l HEPES buffer. SP staining was performed as for the cell lines.

Cells were then analysed using FACS Aria (Becton Dickinson, San Jose, CA, USA). The Hoechst 33342 dye was excited at $357 \mathrm{~nm}$ and its fluorescent emission was measured at Hoechst blue fluorescence and Hoechst red fluorescence. SP cells and mature plasma cells (i.e., CD138-positive non-SP cells) were then sorted accordingly (Fig 1). 


\section{Aldefluor assay by flow cytometry}

Aldefluor assay was performed according to the manufacturer's instruction (Stem Cell Technologies, Vancouver, BC, Canada). The aldehyde dehydrogenase (ALDH) high and low populations of RPMI8226 cells were analysed and sorted with the FACS Aria.

\section{cDNA synthesis}

cDNA from sorted cells was synthesized with the WT-Ovation RNA Amplification System (Nugen, San Carlos, CA, USA) according to the user's guide. The cDNA concentration was estimated using the NanoDrop ND-1000 spectrophotometer (Thermo Scientific, Wilmington, DE, USA).

\section{CTA gene selection}

Cancer testis antigens expression in various types of cancers should be different. In order to identify CTA signatures in MM among different stage MM patients, we used the open access microarray database: Mayo Clinic MM Microarray. This database was established by analysing BM samples of 91 new, 23 smouldering and 26 relapsed MM patients from MM research consortium (MMRC, http://www.broadinstitute.org/mmgp/home). Then, we integrated this database with the CTA gene bank database (http://www.cta.lncc.br/) to specifically study the expression of CTA genes in MM patients and identified 90 CTA genes expressed by the majority of MM patients (Fig 2A).

\section{Primers and qRT-PCR}

We designed primers for these 90 CTA and 5 housekeeping genes (data not shown) using Primer 3 software and selected those that contained at least 1 exon-exon junction to reduce the genomic DNA contamination. One pair of primers for each gene was added to each well of a 96-well plate to form the assay, comprising 90-CTA genes and 5 housekeeping genes, and one well as a blank control without any primer. qRT-PCR analysis of the gene expression was then performed using $\mathrm{RT}^{2}-\mathrm{SYBR}^{\circledR}$ Green PCR Master Mix (Qiagen, Valencia, CA, USA) with 40 cycles of $15 \mathrm{~s}$ at $95^{\circ} \mathrm{C}$ and $1 \mathrm{~min}$ at $58^{\circ} \mathrm{C}$ on an ABI 7500 RealTime PCR System (Applied Biosystems, Foster City, CA, USA). Fluorescence data were collected at $58^{\circ} \mathrm{C}$ after each cycle. After the final cycle, melting curve analysis of all samples was conducted within the range of $58-95^{\circ} \mathrm{C}$. The specificity of the PCR products was verified by the targeted product size using gel electrophoresis and melting curve analysis. The threshold cycle and $2^{-\Delta \Delta C_{t}}$ method were used for calculating the relative amount of the target RNA using the average of the five house-keeping genes as internal control, according to user's manual. The experiments were repeated in triplicate.

\section{Immunoglobulin heavy chain (IGH) gene rearrangement analysis}

DNA was isolated from cells using the QIAmp system (Qiagen). The concentration of DNA was estimated using the NanoDrop ND-1000 spectrophotometer. DNA quality was assessed using Agilent 2100 Bioanalyser (Agilent Technologies, Santa Clara, CA, USA). PCR primer sequences for $I G H V$ gene framework region 3 (FR3) were designed as previously reported (Kummalue et al, 2010). PCR of the $I G H$ genes to assess clonality used the BIOMED-2 system. Master mixes were purchased from Invivoscribe Technologies (San Diego, CA, 
USA), and the PCR was done as per manufacturer's instructions and used HotStart Taq DNA polymerase (Qiagen) (Burack et al, 2010).

\section{Immunofluorescence}

SP and non-SP (NSP) cells were sorted with Hoechst 33342 staining. After washing, cells were resuspended in phosphate-buffered saline (PBS) and transferred to a coverslip coated with poly-L-lysine. After a 1-h incubation at room temperature, excess cell suspension was removed. Cells were washed, fixed, blocked with PBS containing $0.1 \%$ bovine serum albumin, and incubated with primary anti-AURKA antibody (Thermo Fisher Scientific) for $1 \mathrm{~h}$. Subsequently, cells were washed with PBS and incubated with TXRed-labelled secondary antibody. After washing, coverslips were mounted on slides in fluorescent mounting medium containing 4',6-diamidino-2-phenylindole (DAPI) (ProLong antifade reagent with DAPI; Invitrogen, Carlsbad, CA, USA). Fluorescence was detected using an Olympus microscope and images were acquired using Cellsens Dimension software.

\section{SiRNA knock down}

LUZP4 gene siRNA and scrambled siRNA (Santa Cruz Biotechnology, Dallas, TX, USA) were used at $100 \mathrm{nmol} / \mathrm{l}$ to transfect RPMI8226 cells using Lipofectamine RNAiMAX (Invitrogen), as previously reported (Wen et al, 2008). Knockdown efficiency of siRNAs was confirmed by Western blot analysis.

\section{Western blotting}

Cell lysates were prepared and analysed by Western blot as described previously. The membrane was probed with antibody to LUZP4 (Santa Cruz Biotechnology). After detection, the membrane was completely stripped and ACTB ( $\beta$-actin) was used as loading control (Wen et al, 2008).

\section{Cell colony assay}

A soft agar colony assay was performed as previously reported (Wen et al, 2011). Briefly, $1.5 \mathrm{ml}$ base agar layers of $0.6 \%$ agarose were prepared in $35 \mathrm{~mm}$ dishes by combining equal volumes of $1.2 \%$ low melting temperature agarose (Thermo Fisher Scientific) and $2 \times$ RPMI 1640 medium $+20 \%$ FBS $+2 \times$ antibiotics. Next, $5 \times 10^{3}$ cells were resuspended in $0.75 \mathrm{ml}$ $2 \times$ RPMI 1640 medium $+20 \%$ FBS $+2 \times$ antibiotics and mixed with $0.75 \mathrm{ml}$ volumes of $0.6 \%$ agar, then immediately plated on top of base agar. Complete medium was added on the top and changed twice a week. After 2 weeks, dishes were stained with methylene blue, pictures were taken under a phase contrast microscope, and colony number was counted.

\section{Flow cytometric analysis of apoptosis}

Cells were treated with arsenic trioxide (Sigma-Aldrich Co.) or Bortezomib (Millennium

Pharmaceuticals, Cambridge, MA, USA) for $24 \mathrm{~h}$. Dual staining with Annexin V-fluorescein isothiocyanate (FITC) and PI (Propidium iodide) (Becton Dickinson) was used to detect apoptosis according to the manufacturer's instructions as previously reported (Wen $e t a l$, 2008, 2010). 


\section{Statistical analysis}

Statistical analysis was conducted with the SPSS 10 SPSS Inc., Chicago, IL, USA), using $t$ test or 1-way analysis of variance (ANova) where appropriate.

\section{Results}

\section{SP cells from myeloma patients containing clonal population}

The flow cytometric analysis revealed that in primary MM patient BM, SP cells are CD138negative and NSP cells are CD138+ (Fig 1A, B). Immunoglobulin heavy chain $(I G H)$ rearrangement study showed that SP and MPC from myeloma patients possess the identical $I G H$ rearrangement pattern (Fig 1C). This result indicates that the SP of these patients contains myeloma stem cells that are of same cell origin of the mature neoplastic plasma cells.

\section{MM-CTA were enriched in SP in MM cell lines}

With the strategy shown in Fig 2A, we designed the MM-specific CTA gene assay composed of 90 CTA genes and 5 housekeeping genes. Among these 90 CTA genes, 5 genes were up-regulated in the new MM patient and 4 genes were up-regulated in the patient with smouldering MM. Futhermore, 17 CTA genes were up-regulated in relapsed MM patient, compared with normal control (Fig 2B). The expression levels as well as the ratio to normal control in relapsed MM, smouldering MM, and new MM are shown in Table I.

To test if CTA gene expression is enriched in MM SP, we investigated the CTA expression profile in various myeloma cell lines and primary samples. We collected SP and MPC, respectively, from MM cells lines including RPMI8226, MM1S and U266. Using a threshold of $>2$-fold change for genes expression (SP: MPC) and $P$ value $<0.05$ after $t$-test, 16 CTA genes were up-regulated in RPMI8226 (Fig 3A), 13 CTA genes in MM1S (Fig 3B) and 11 CTA genes in U266 cell line (Fig 3C). Of note, among these genes, GAGE12F, MAGEA4, MAGEA5, SSX1, and TOP2A genes showed overlap in 2 of 3 cell lines, and $L U Z P 4$ and $O D F 1$ were universally up-regulated in all $3 \mathrm{MM}$ cell lines. Furthermore, Immunofluorescence membrane staining with anti-CTA antigen AURKA antibody was performed. In agreement with our PCR data, the AURKA antibody stain showed higher membrane expression in SP cells than in MPC (Figure S1).

\section{MM-CTA were enriched in SP in MM BM samples}

We further extended our study to primary MM cells. Nineteen CTA genes were significantly up-regulated in SP (fold change $>2$ compared with MPC, $P<0.05$, $t$-test); Fig 4A.

Interestingly, AURKA, DDX43, FANCI, MAGEA3, TEX14, and LUZP4 were also identified as up-regulated genes in the SP of at least $1 \mathrm{MM}$ cell line. In addition, 3 CTA genes (AKAP4, MAGEA3, and SSX2) and 7 genes (ANKRD45, FANCI, LUZP4, MAGEA12, $M A G E B 3, R R M 2$, and TEX14) were over-expressed by the SP of 8/8 and 7/8 MM patients, respectively (Table II). The detailed information for MM patients and fold-change of CTA gene in each patient are shown in Tables SI and SII, respectively. 
In contrast to MM BM, only 2 CTA genes, DUT and KDM5B (JARIDIB), showed moderate increase (2.2- and 4.5-fold, respectively) in the SP of normal BM compared with MPC (Fig 4B). Of interest, these 2 genes were not up-regulated in the SP of MM patients.

Additionally, except for AKAP4 (3.95-fold), CFLAR (2.93-fold), HISTIH2BG (2.24-fold), $M A G E B 2$ (4.18-fold) and MAGEB3 (3.97-fold), the expression level of 19 up-regulated CTA genes in SP of MM BM was more than 5 times higher than that in SP of normal BM control (Fig 4C).

It should be noted that the SP cells in the myeloma patients include both cancer stem cells and normal haematopoietic stem cells (HSC) while the SP cells in normal samples contain only normal HSC (Morita et al, 2006; Golebiewska et al, 2011). Thus, the differences of CTA expression between the SP cells of myeloma patients and controls are most probably due to the increased expression of CTA genes of cancer stem cells within the SP cells of myeloma patients.

All these results suggested that CTA have unique expression profiles in the SP of MM cells, suggesting that CTA may serve as targets for immunotherapy that is specific for MM stem cells, which may lead to the long term cure of MM.

\section{LUZP4 gene expression is required for colony formation and drug resistance}

To explore the function of the gene LUZP4, which was universally up-regulated in SP of MM cell lines and primary MM BM, we used siRNA to knockdown gene expression and the efficiency was confirmed by Western blot (Fig 5A). Given its up-regulated expression level in MM stem cells, which play a critical role in tumour development, disease recurrence, resistance and chemotherapy (Huff \& Matsui, 2008; Matsui et al, 2008; Brennan \& Matsui, 2009; Ghosh \& Matsui, 2009; Jakubikova et al, 2011; Paino et al, 2012), we hypothesized that the function of $L U Z P 4$ may be related to colony forming ability and drug resistance. Firstly, the LUZP4 siRNA knocked-down U266 cells and control cells were grown on agar for 2 weeks: the results showed that $L U Z P 4$ knocked-down cells formed fewer colonies compared to control cells. Next, we treated the LUZP4 knocked-down U266 cells with arsenic trioxide and bortezomib for $24 \mathrm{~h}$ and the results showed that LUZP4 knocked-down U266 cells were more sensitive to chemotherapy.

\section{Discussion}

Our results have indicated that certain CTA genes (e.g., AURKA, DDX43, FANCI, MAGEA3, TEX14 and LUZP4) are upregulated in both SP cells of the majority of MM cell lines and primary marrow samples from MM patients compared to the mature myeloma cells. This finding indicates that upregulation of CTA genes is a common feature for myeloma cells with stem cell features. This is in accordance with previous reports showing that enhanced expression of CTA genes in glioma stem cells and that CTA genes promoters may be tightly regulated by methylations and the methylation levels in promoter regions in cancer stem cells are lower than that in differentiated cells (Yawata et al, 2010). This mechanism may have contributed to the up-regulation of CTA in cancer stem cells in general and in myeloma stem cells in our study. 
In this study, we used SP cells identified by flow cytometry, based on the unique property of stem cells, which pump out Hoechst 33342 dye due to their high expression of ATP binding cassette transporters, to identify myeloma stem cells in MM. This is because studies have demonstrated that SP cells exhibited clonogenic and tumourigenic potential in MM (Jakubikova et al, 2011; Ikegame et al, 2012). We did not use surface markers, another commonly used approach to study cancer stem cells, because the surface marker phenotype for myeloma stem cells remains controversial (Yaccoby \& Epstein, 1999; Matsui et al, 2008; Jakubikova et al, 2011). Similar to the previous study (Jakubikova et al, 2011), we demonstrated an identical $I G H$ gene rearrangement pattern in the SP cells and mature neoplastic plasma cells (Fig 1), confirming that our methodology is adequate to identify the myeloma stem cells from marrow samples of myeloma patients. In contrast, clonal IGH rearrangement was absent in the SP and mature plasma cells of controls when using an identical approach, indicating a lack of clonal populations (data not shown). Additionally, we used another function assay, ALDEFLUOR, for identifying and isolating stem cells of a myeloma cell line (RPMI8226) based on the high expression of aldehyde dehydrogenase of stem cells (Matsui et al, 2008; Brennan et al, 2010). The stem cells isolated using this functional assay showed similarly elevated CTA expression as isolated by SP cells, confirming that the overexpression of CTA is unique character of myeloma stem cells (Figure S2).

Of the CTA genes up-regulated among the SP of MM, LUZP4, MAGEA12, MAGEA3, $M A G E B 2, M A G E B 3$, and $S S X 2$, have particular potential for consideration as targets for immunotherapy targeting the myeloma stem cell-like cells. These CTA genes are localized to the X-chromosome (Table II). As shown by previous studies (Dakshinamurthy et al, 2008), CTA genes localized to this chromosome are highly immunogenic; in contrast, immunogenicity of CTA localized on autosomal chromosomes has not been proven (Scanlan et al, 2004).

It is worth noting that this study included $8 \mathrm{MM}$ patients (Tables SI, SII); however, it remains difficult to link CTA gene expression profile to the different stages of MM. We believe that more MM cases are required to shed the light on this task.

Immunotherapy, targeting myeloma stem cell-like cells expressing CTA, is particularly attractive for MM patients who have achieved complete remission. The majority, if not all, of these patients developed recurrent disease that is resistant to multiple agents. It has been proposed that the myeloma stem cells, which are highly drug-resistant in nature and have self-renewal capability, play a critical role in the recurrence of MM. This immunotherapy approach is likely to eradicate the myeloma stem cells in these patients to ensure long-term remission of these patients. In our previous studies, we have demonstrated the feasibility of this approach and showed that cytotoxic T-cells (CTL) specific for PRAME-derived peptide can target leukaemic and leukaemic-precursor cells expressing PRAME (Quintarelli et al, 2011).

Additionally, previous studies have suggested that some CTA genes may function as regulators of cell-cycle progression, apoptosis and transcriptional repression (Scanlan et al, 2002; Yawata et al, 2010). CTA genes may thus participate in maintaining the stem cell pool 
directly or indirectly. In the present study, knock down LUZP4 expression with siRNA reduced the colony-forming ability of MM cells and sensitized the cells to chemotherapy. This finding will initiate further investigation regarding the function of CTA genes in MM.

In summary, the current study identifies a subset of CTA genes highly expressed by multiple myeloma stem cell-like side population cells using a unique high throughput qRT-PCR assay designed in our laboratory. The resulting data provides the framework for using immunotherapy to target the myeloma stem cells expressing high levels of CTA for the long-term cure of MM.

\section{Supplementary Material}

Refer to Web version on PubMed Central for supplementary material.

\section{Acknowledgments}

Funding

This work was supported by grants from the National Institutes of Health, USA (R33CA173382 to YZ, and R21/ RCA150109A to JW and CC).

\section{References}

Borrello I. Can we change the disease biology of multiple myeloma? Leukemia Research. 2012; 36(Suppl. 1):S3-S12. [PubMed: 23176722]

Brennan SK, Matsui W. Cancer stem cells: controversies in multiple myeloma. Journal of Molecular Medicine (Berlin). 2009; 87:1079-1085.

Brennan SK, Wang Q, Tressler R, Harley C, Go N, Bassett E, Huff CA, Jones RJ, Matsui W. Telomerase inhibition targets clonogenic multiple myeloma cells through telomere lengthdependent and independent mechanisms. PLoS One. 2010; 5:e12487. [PubMed: 20824134]

van der Bruggen P, Traversari C, Chomez P, Lurquin C, De Plaen E, Van den Eynde B, Knuth A, Boon T. A gene encoding an antigen recognized by cytolytic T lymphocytes on a human melanoma. Science. 1991; 254:1643-1647. [PubMed: 1840703]

Burack WR, Laughlin TS, Friedberg JW, Spence JM, Rothberg PG. PCR assays detect B-lymphocyte clonality in formalin-fixed, paraffin-embedded specimens of classical hodgkin lymphoma without microdissection. American Journal of Clinical Pathology. 2010; 134:104-111. [PubMed: 20551274]

de Carvalho F, Vettore AL, Colleoni GW. Cancer/Testis Antigen MAGE-C1/CT7: new target for multiple myeloma therapy. Clinical \& Developmental Immunology. 2012; 2012:257695. [PubMed: 22481966]

Chanan-Khan AA, Borrello I, Lee KP, Reece DE. Development of target-specific treatments in multiple myeloma. British Journal of Haematology. 2010; 151:3-15. [PubMed: 20618339]

Cruz RD, Tricot G, Zangari M, Zhan F. Progress in myeloma stem cells. American Journal of Blood Research. 2011; 1:135-145. [PubMed: 22432075]

Dakshinamurthy AG, Ramesar R, Goldberg P, Blackburn JM. Infrequent and low expression of cancer-testis antigens located on the $\mathrm{X}$ chromosome in colorectal cancer: implications for immunotherapy in South African populations. Biotechnology Journal. 2008; 3:1417-1423. [PubMed: 18956367]

Ghosh N, Matsui W. Cancer stem cells in multiple myeloma. Cancer Letters. 2009; 277:1-7. [PubMed: 18809245]

Golebiewska A, Brons NH, Bjerkvig R, Niclou SP. Critical appraisal of the side population assay in stem cell and cancer stem cell research. Cell Stem Cell. 2011; 8:136-147. [PubMed: 21295271] 
Hajek R, Okubote SA, Svachova H. Myeloma stem cell concepts, heterogeneity and plasticity of multiple myeloma. British Journal of Haematology. 2013; 163:551-564. [PubMed: 24111932]

Huff CA, Matsui W. Multiple myeloma cancer stem cells. Journal of Clinical Oncology. 2008; 26:2895-2900. [PubMed: 18539970]

Ikegame A, Ozaki S, Tsuji D, Harada T, Fujii S, Nakamura S, Miki H, Nakano A, Kagawa K, Takeuchi K, Abe M, Watanabe K, Hiasa M, Kimura N, Kikuchi Y, Sakamoto A, Habu K, Endo M, Itoh K, Yamada-Okabe H, Matsumoto T. Small molecule antibody targeting HLA class I inhibits myeloma cancer stem cells by repressing pluripotency-associated transcription factors. Leukemia. 2012; 26:2124-2134. [PubMed: 22430632]

Jakubikova J, Adamia S, Kost-Alimova M, Klippel S, Cervi D, Daley JF, Cholujova D, Kong SY, Leiba M, Blotta S, Ooi M, Delmore J, Laubach J, Richardson PG, Sedlak J, Anderson KC, Mitsiades CS. Lenalidomide targets clonogenic side population in multiple myeloma: pathophysiologic and clinical implications. Blood. 2011; 117:4409-4419. [PubMed: 21321360]

Jemal A, Siegel R, Ward E, Murray T, Xu J, Thun MJ. Cancer statistics, 2007. CA: A Cancer Journal for Clinicians. 2007; 57:43-66. [PubMed: 17237035]

Kummalue T, Chuphrom A, Sukpanichanant S, Pongpruttipan T. Detection of monoclonal immunoglobulin heavy chain gene rearrangement (FR3) in Thai malignant lymphoma by High Resolution Melting curve analysis. Diagnostic Pathology. 2010; 5:31. [PubMed: 20482846]

Matsui W, Wang Q, Barber JP, Brennan S, Smith BD, Borrello I, McNiece I, Lin L, Ambinder RF, Peacock C, Watkins DN, Huff CA, Jones RJ. Clonogenic multiple myeloma progenitors, stem cell properties, and drug resistance. Cancer Research. 2008; 68:190-197. [PubMed: 18172311]

Mengus C, Schultz-Thater E, Coulot J, Kastelan Z, Goluza E, Coric M, Spagnoli GC, Hudolin T. MAGE-A10 cancer/testis antigen is highly expressed in high-grade non-muscle-invasive bladder carcinomas. International Journal of Cancer. 2013; 132:2459-2463.

Morita Y, Ema H, Yamazaki S, Nakauchi H. Non-side-population hematopoietic stem cells in mouse bone marrow. Blood. 2006; 108:2850-2856. [PubMed: 16804114]

Munshi NC, Tricot G, Desikan R, Badros A, Zangari M, Toor A, Morris C, Anaissie E, Barlogie B. Clinical activity of arsenic trioxide for the treatment of multiple myeloma. Leukemia. 2002; 16:1835-1837. [PubMed: 12200700]

Paino T, Ocio EM, Paiva B, San-Segundo L, Garayoa M, Gutierrez NC, Sarasquete ME, Pandiella A, Orfao A, San Miguel JF. CD20 positive cells are undetectable in the majority of multiple myeloma cell lines and are not associated with a cancer stem cell phenotype. Haematologica. 2012; 97:1110-1114. [PubMed: 22315496]

Quintarelli C, Dotti G, Hasan ST, De Angelis B, Hoyos V, Errichiello S, Mims M, Luciano L, Shafer J, Leen AM, Heslop HE, Rooney CM, Pane F, Brenner MK, Savoldo B. High-avidity cytotoxic T lymphocytes specific for a new PRAME-derived peptide can target leukemic and leukemicprecursor cells. Blood. 2011; 117:3353-3362. [PubMed: 21278353]

Richardson PG, Barlogie B, Berenson J, Singhal S, Jagannath S, Irwin D, Rajkumar SV, Srkalovic G, Alsina M, Alexanian R, Siegel D, Orlowski RZ, Kuter D, Limentani SA, Lee S, Hideshima T, Esseltine DL, Kauffman M, Adams J, Schenkein DP, Anderson KC. A phase 2 study of bortezomib in relapsed, refractory myeloma. New England Journal of Medicine. 2003; 348:26092617. [PubMed: 12826635]

Scanlan MJ, Gure AO, Jungbluth AA, Old LJ, Chen YT. Cancer/testis antigens: an expanding family of targets for cancer immunotherapy. Immunological Reviews. 2002; 188:22-32. [PubMed: 12445278]

Scanlan MJ, Simpson AJ, Old LJ. The cancer/testis genes: review, standardization, and commentary. Cancer Immunity. 2004; 4:1. [PubMed: 14738373]

Wen J, Cheng HY, Feng Y, Rice L, Liu S, Mo A, Huang J, Zu Y, Ballon DJ, Chang CC. P38 MAPK inhibition enhancing ATO-induced cytotoxicity against multiple myeloma cells. British Journal of Haematology. 2008; 140:169-180. [PubMed: 18173754]

Wen J, Feng Y, Huang W, Chen H, Liao B, Rice L, Preti HA, Kamble RT, Zu Y, Ballon DJ, Chang CC. Enhanced anti-myeloma cytotoxicity by the combination of arsenic trioxide and bortezomib is further potentiated by p38 MAPK inhibition. Leukemia Research. 2010; 34:85-92. [PubMed: 19608275] 
Wen J, Feng Y, Bjorklund CC, Wang M, Orlowski RZ, Shi ZZ, Liao B, O’Hare J, Zu Y, Schally AV, Chang CC. Luteinizing Hormone-Releasing Hormone (LHRH)-I antagonist cetrorelix inhibits myeloma cell growth in vitro and in vivo. Molecular Cancer Therapeutics. 2011; 10:148-158. [PubMed: 21062912]

Yaccoby S, Epstein J. The proliferative potential of myeloma plasma cells manifest in the SCID-hu host. Blood. 1999; 94:3576-3582. [PubMed: 10552969]

Yawata T, Nakai E, Park KC, Chihara T, Kumazawa A, Toyonaga S, Masahira T, Nakabayashi H, Kaji T, Shimizu K. Enhanced expression of cancer testis antigen genes in glioma stem cells. Molecular Carcinogenesis. 2010; 49:532-544. [PubMed: 20082319] 
(B) SP

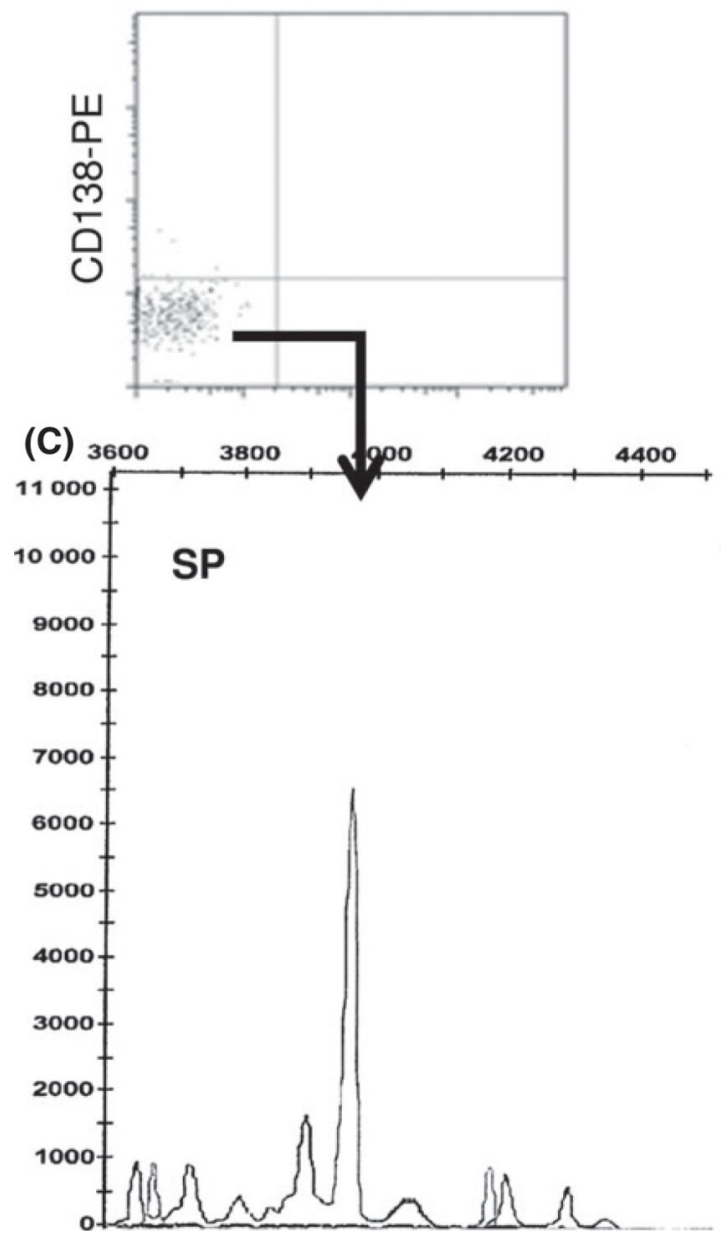

(A) MM patient
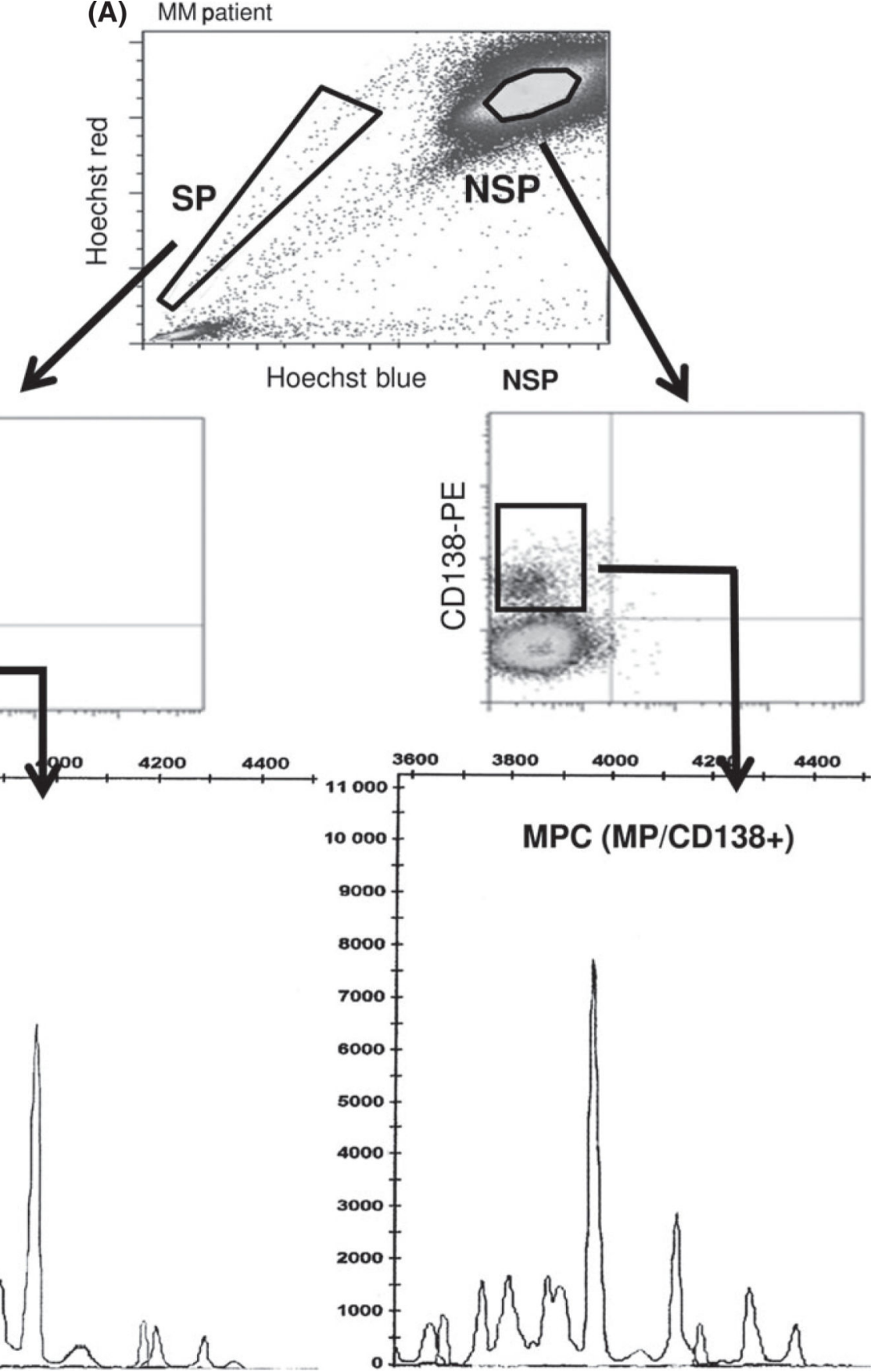

Fig 1.

Side population in primary MM BM sample. (A) Primary MM BM sample was subjected to Hoechst 33342 SP staining. The SP and non-SP (NSP) are shown. (B) SP and NSP populations were analysed for CD138 expression. (C) IGH rearrangement assay was performed in SP and MPC (NSP/CD138+). The experiments were repeated three times, and representative results are shown. MM, multiple myeloma; $\mathrm{BM}$, bone marrow; SP, side population; NSP, non-SP; MPC, mature plasma cells. 
(A)

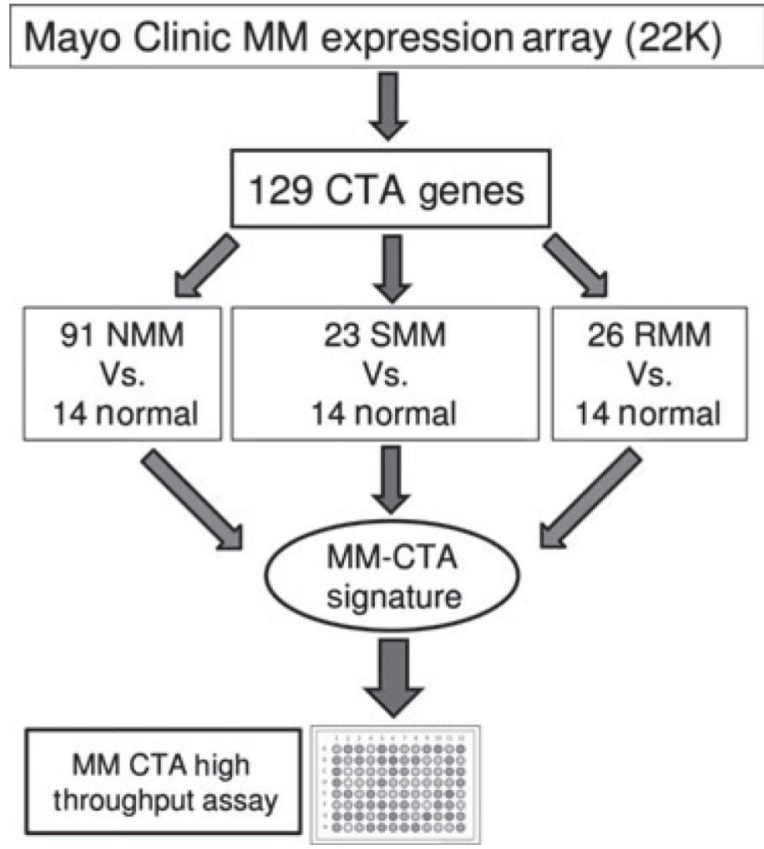

(B)

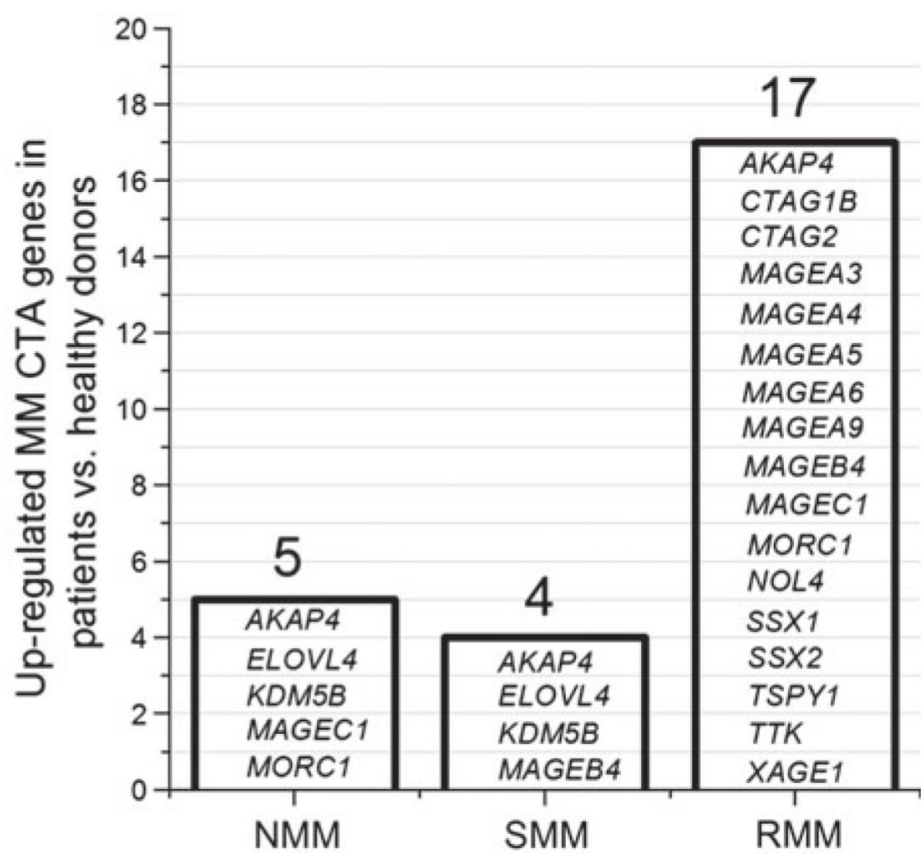

Fig 2.

Design of the MM-specific CTA gene high throughput qRT-PCR Assay. (A) Cancer testis antigen (CTA) gene expression data from Mayo Clinic multiple myeloma (MM) expression array was compared among four groups: new MM patients (NMM), smouldering MM (SMM), relapsed MM (RMM) and normal donors by 1-way anova. If the fold change was $>2$ (MM: normal) and $P$ value $<0.05$, the gene was defined as an abnormal up-regulated gene. (B) Highly expressed CTA genes in NMM, SMM, and RMM groups compared with healthy donor are shown. 
(A)

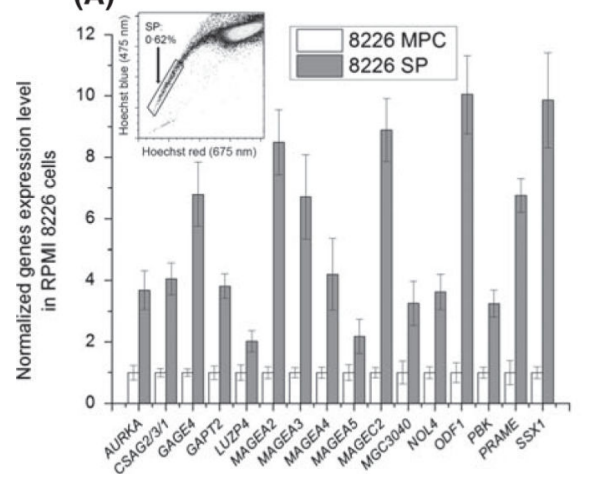

(B)

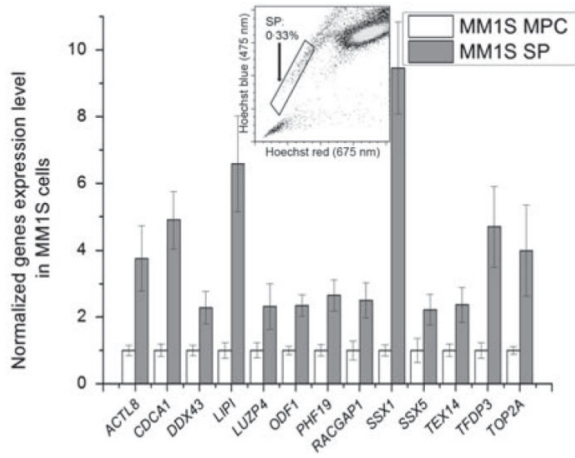

(C)

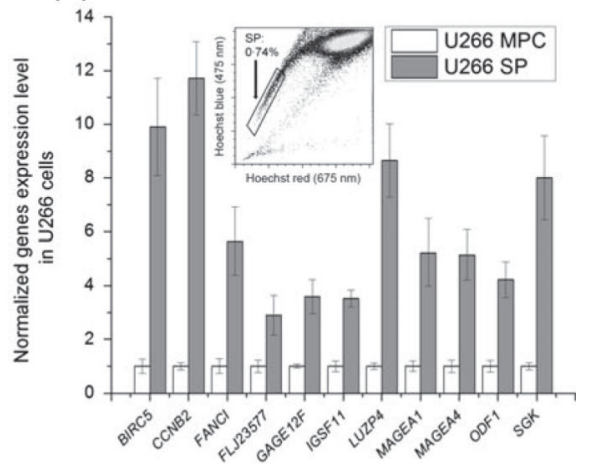

Fig 3.

Up-regulated CTA genes in the SP of MM cell lines compared with MPC. SP and MPC cells were sorted from three MM cell lines and qRT-PCR-based CTA gene assay was carried out. Up-regulated CTA genes in SP (fold change $>2$ compared with MPC cells, $P<0.05$ after $t$ test) are shown. Data are presented as mean \pm standard deviation of three independent experiments after normalizing the data to the MPC. The flow cytometric analysis of SP cells in each cell line is shown as inset. (A) MM cell line RPMI8226. (B) MM cell line MM1S. (C) MM cell line U266. CTA, cancer testis antigen; MM, multiple myeloma; BM, bone marrow; SP, side population; MPC, mature plasma cells. 

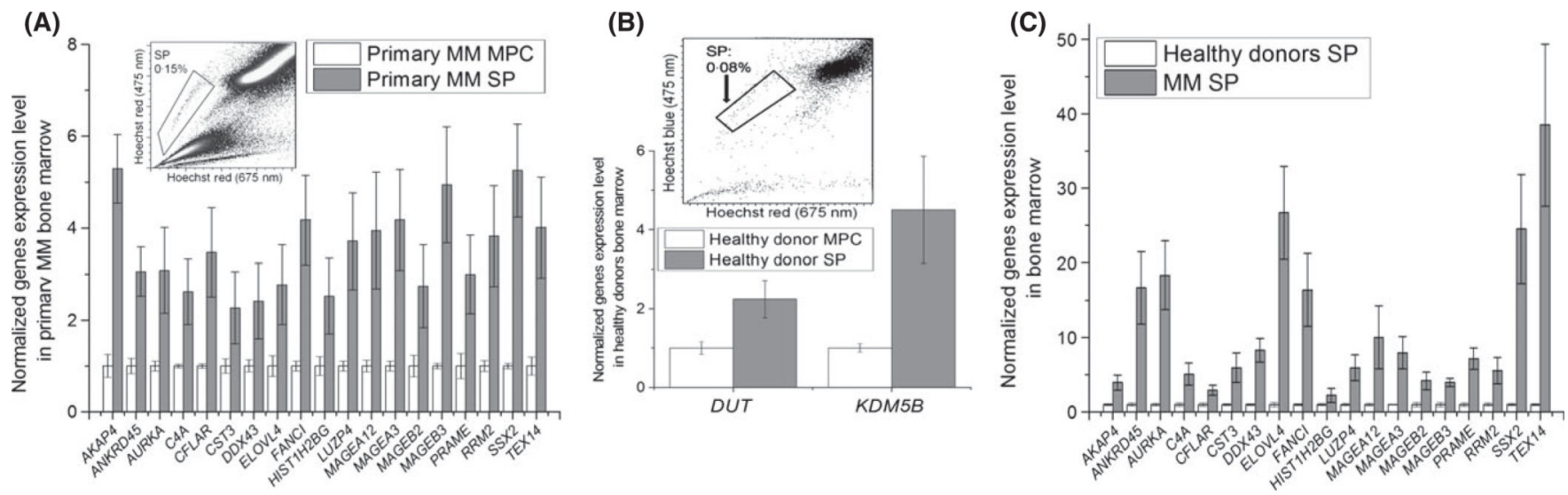

Fig 4.

Up-regulated CTA genes in the SP of primary MM and healthy donor BM compared with MPC. SP and MPC were sorted out from BM of MM and healthy donors and qRT-PCR based CTA gene assay was carried out. (A) Up-regulated CTA genes in 8 MM patient SP cells are shown (fold change $>2$ compared with MPC, $P<0.05$ after $t$-test). Data are presented as mean of eight MM samples (each has triplicates) \pm standard deviation (SD) after normalizing the data to the MPC. The flow cytometric analysis of SP cells is shown as inset. (B) Up-regulated CTA genes in four healthy donor SP cells are shown (fold change >2 compared with MPC, $P<0.05$ after $t$-test). Data are presented as mean of four control samples (each has triplicates) \pm SD after normalizing the data to the MPC. The flow cytometric analysis of SP cells is shown as inset. (C) The up-regulated CTA genes in SP of primary MM BM have much lower expression level in SP of healthy donor BM. CTA, cancer testis antigen; MM, multiple myeloma; $\mathrm{BM}$, bone marrow; SP, side population; MPC, mature plasma cells. 


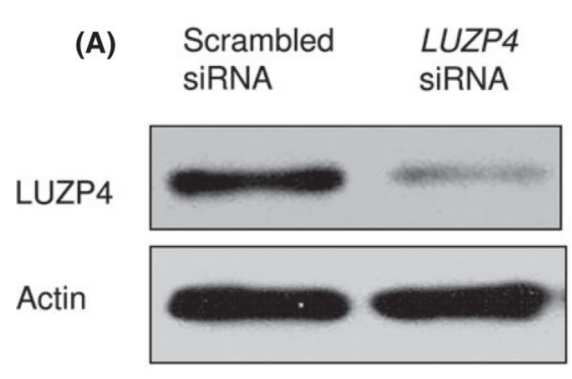

(B)

\section{Scrambled LUZP4 SiRNA}
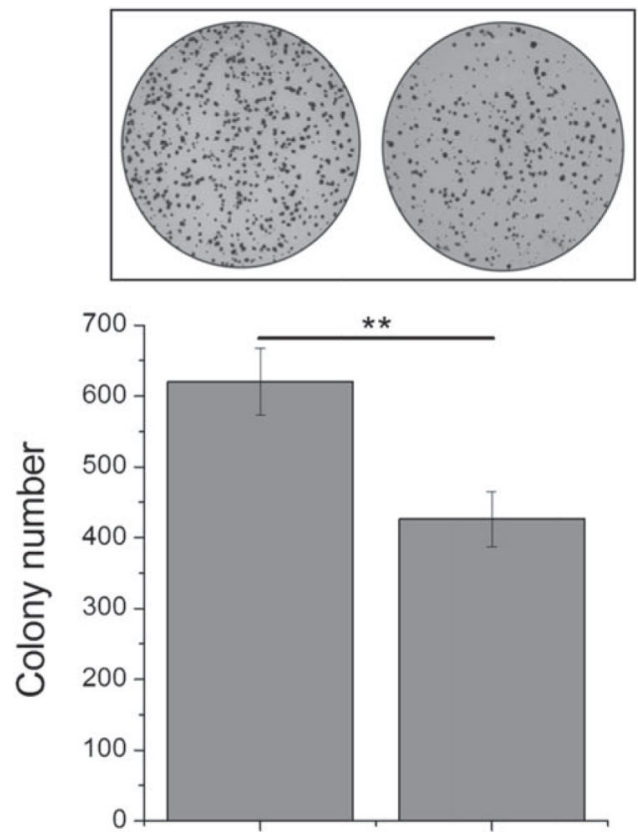

(C)
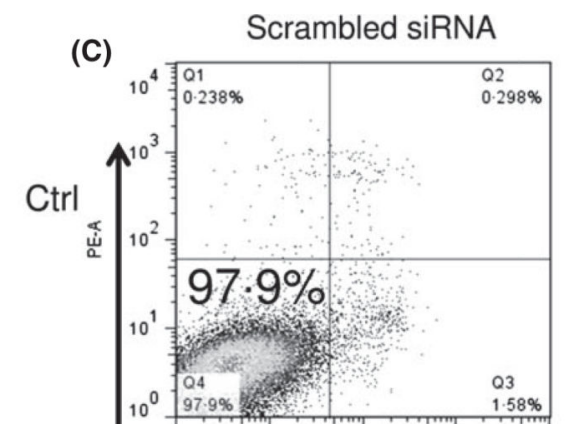

Fig 5.

LUZP4 is related with the colony-forming ability and drug resistance in MM cells. (A)

LUZP4 expression in MM cell line U266 was knocked down with siRNA and the efficiency was verified with Western blot. The experiments were repeated three times, and representative figures are shown. (B) Colony formation assay. $5 \times 103$ U266 knock down with LUZP4 siRNA or scrambled siRNA were resuspended in agar and seeded in a 6-well plate. Complete medium was added on the top and changed twice a week. After 2 weeks, dishes were stained with methylene blue, pictures were taken under a phase contrast microscope, and colony number was counted. Representative images are shown from independent experiments (upper panel), and data are presented as mean \pm standard deviation of triplicate experiments (**P<0.01, $t$-test) (lower panel). (C) Apoptosis assay. U266 knock down with LUZP4 siRNA or scrambled siRNA were seeded in 6-well plate with complete medium. Cells were treated with $40 \mathrm{nmol} / \mathrm{l}$ bortezomib (BZM) or $2 \mu \mathrm{mol} / \mathrm{l}$ arsenic trioxide (ATO) for $24 \mathrm{~h}$ followed with flow cytometric apoptosis assay. The percentage of Annexin V/PI double-negative cells was noted. Experiments were repeated three times, and representative blots are shown. 


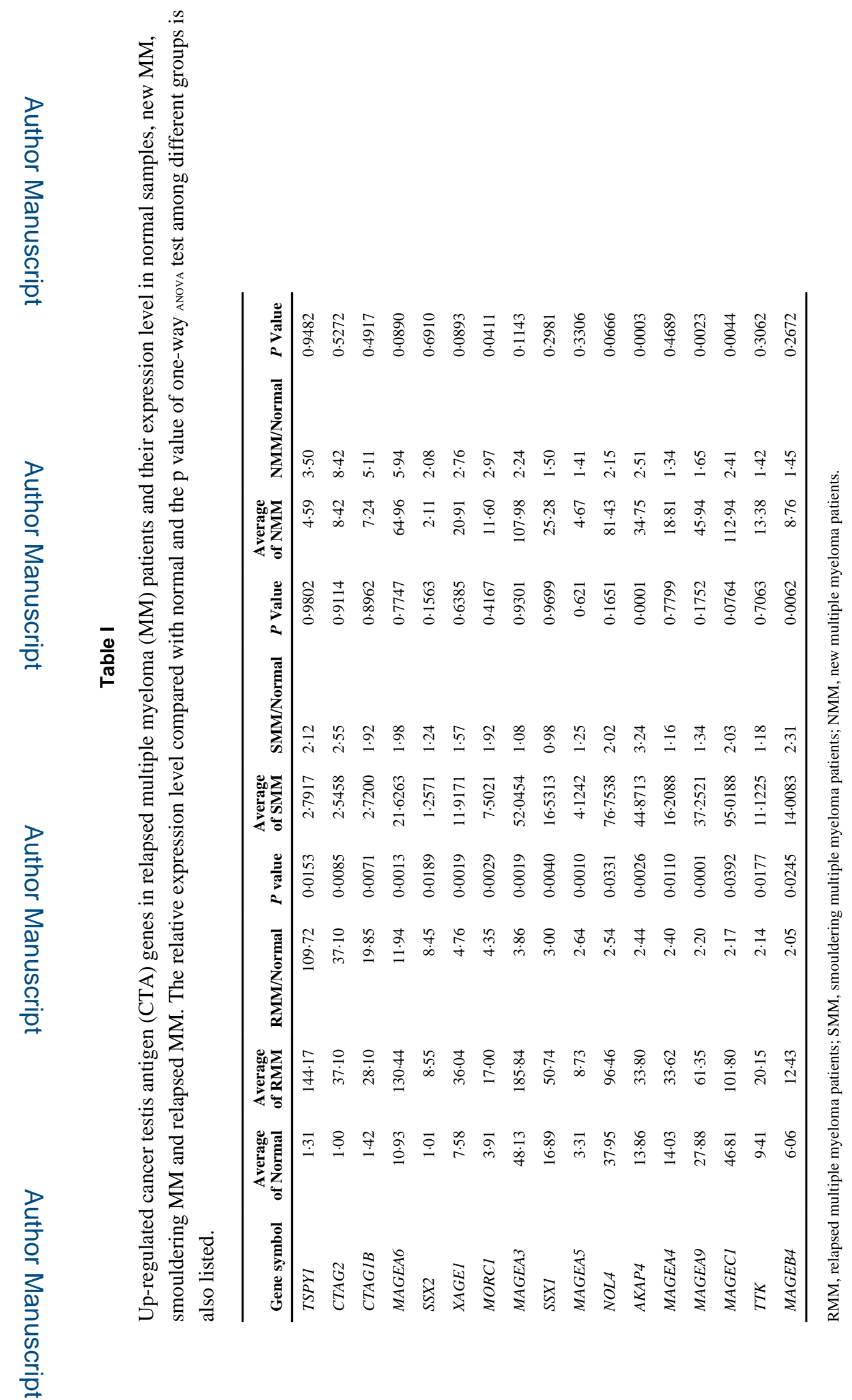

Br J Haematol. Author manuscript; available in PMC 2015 March 19. 


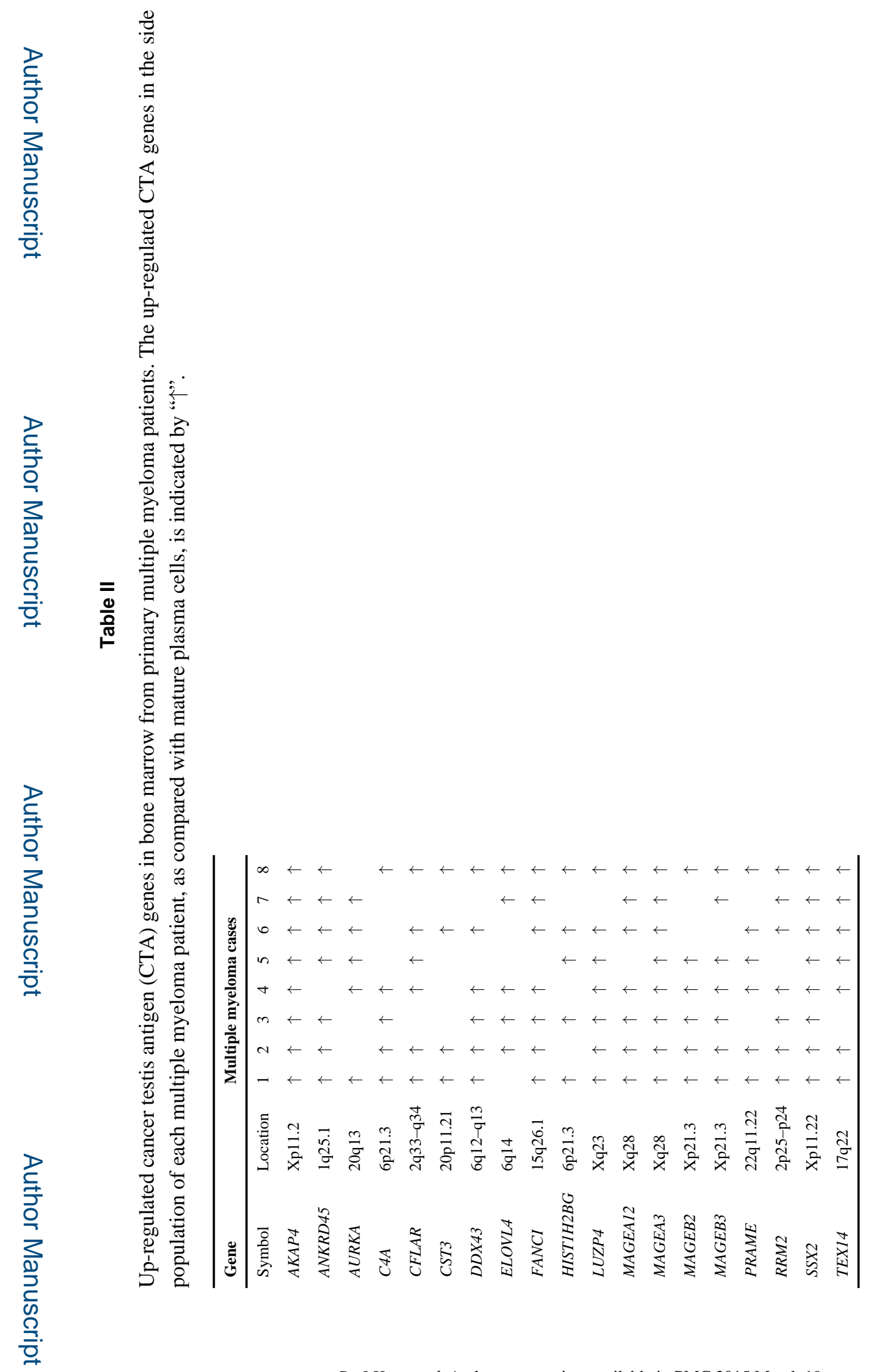

Br J Haematol. Author manuscript; available in PMC 2015 March 19. 